\title{
Reduction of heparan sulphate-associated anionic sites in the glomerular basement membrane of rats with streptozotocin- induced diabetic nephropathy
}

\author{
J. van den Born ${ }^{1}$, A. A. van Kraats ${ }^{1}$, M. A.H. Bakker ${ }^{1}$, K. J. M. Assmann ${ }^{2}$, H. B.P. M. Dijkman ${ }^{2}$, \\ J. A. W. M. van der Laak ${ }^{2}$, J.H.M.Berden ${ }^{1}$ \\ ${ }^{1}$ Department of Nephrology, University Hospital St. Radboud, Nijmegen, The Netherlands \\ ${ }^{2}$ Department of Pathology, University Hospital St. Radboud, Nijmegen, The Netherlands
}

\begin{abstract}
Summary Heparan sulphate-associated anionic sites in the glomerular basement membrane were studied in rats 8 months after induction of diabetes by streptozotocin and in age- and sex-matched control rats, employing the cationic dye cuprolinic blue. Morphometric analysis at the ultrastructural level was performed using a computerized image processor. The heparan sulphate specificity of the cuprolinic blue staining was demonstrated by glycosaminoglycan-degrading enzymes, showing that pretreatment of the sections with heparitinase abolished all staining, whereas chondroitinase $\mathrm{ABC}$ had no effect. The majority of anionic sites ( $74 \%$ in diabetic and $81 \%$ in control rats) were found within the lamina rara externa of the glomerular basement membrane. A minority of anionic sites were scattered throughout the lamina densa and lamina rara interna, and were significantly smaller than those in the lamina rara externa of the glomerular basement membrane $(p<0.001$ and $p<0.01$ for diabetic and control rats, respectively). Diabetic rats progressively developed
\end{abstract}

albuminuria reaching $40.3(32.2-62.0) \mathrm{mg} / 24 \mathrm{~h}$ after 8 months in contrast to the control animals $(0.8(0.2-$ $0.9) \mathrm{mg} / 24 \mathrm{~h}, p<0.002)$. At the same time, the number of heparan sulphate anionic sites and the total anionic site surface (number of anionic sites $\times$ mean anionic site surface) in the lamina rara externa of the glomerular basement membrane was reduced by $19 \%(p<0.021)$ and by $26 \%(p<0.02)$, respectively. Number and total anionic site surface in the remaining part of the glomerular basement membrane (lamina densa and lamina rara interna) were not significantly changed. We conclude that in streptozotocin-diabetic rats with an increased urinary albumin excretion, a reduced heparan sulphate charge barrier/density is found at the lamina rara externa of the glomerular basement membrane. [Diabetologia (1995) 38: 1169-1175]

Key words Albuminuria, anionic sites, cuprolinic blue, glomerular basement membrane, heparan sulphate, rat, streptozotocin.
Heparan sulphate (HS) is thought to play an important role in the permselective properties of the glomerular capillary wall [1-3], since enzymatic digestion

Received: 12 September 1994 and in final revised form: 22 May 1995

Corresponding author: Dr. J. van den Born, University Hospital St. Radboud, Department of Nephrology, P.O. Box 9101, NL-6500 HB Nijmegen, The Netherlands

Abbreviations: HS, Heparan sulphate; GBM, glomerular basement membrane; HSPG, heparan sulphate proteoglycan; STZ, streptozotocin; LRE, lamina rara externa; LD, lamina densa; LD + LRI, lamina densa + lamina rara interna; ANOVA, analysis of variance. of $\mathrm{HS}$ in the glomerular basement membrane (GBM) causes an increased passage of native ferritin and albumin into the urinary space $[4,5]$. Furthermore, intrarenal or intravenous injection of cationic molecules leads to an increased permeability of the GBM to proteins, due to the neutralization of primarily HS-associated anionic sites of the glomerular capillary wall [6-8]. We have recently demonstrated the induction of an acute selective proteinuria after intravenous injection of a monoclonal antibody against GBM HS [9].

Diabetic nephropathy ultimately develops in 30$40 \%$ of patients suffering from insulin-dependent diabetes mellitus and is initially characterized by micro- 
albuminuria $[10,11]$. The selectivity index of the proteinuria in diabetic patients with microalbuminuria [12-15] suggested a decreased charge-dependent permeability of the GBM. For that reason the significance of GBM heparan sulphate proteoglycan (HSPG) for the development of albuminuria in diabetic nephropathy has been analysed in several studies by quantitation of HSPG or HS in glomeruli or isolated GBM. Studies in humans revealed a decreased content of HS and HSPG within the GBM of kidneys from IDDM patients [16-18]. Investigations have also been performed in experimental models of diabetic nephropathy, mostly in the streptozotocin (STZ)-induced diabetes model in the rat: Biochemical quantitation of HS in the GBM, in glomeruli or in the renal cortex revealed a decreased [1922] or unaltered $[23,24] \mathrm{HS}$ content in rats with STZ-induced diabetic nephropathy. However, these studies do not discriminate between HSPG present in the peripheral GBM, or in the enlarged mesangial matrix that also contains HSPG. An alternative approach is to visualize HS and/or HSPG-core protein by immunohistochemical and histochemical methods. We found, using monoclonal antibodies, a decreased staining for HS in the GBM in kidney biopsies from diabetic patients, which correlated with the fractional protein excretion, whereas the GBM staining for the core protein of HSPG was unaltered [25, 26]. Others, however, found a decreased GBM staining for the core protein of HSPG in diabetic kidneys $[27,28]$. Negatively charged molecules like HS can also be visualized by cationic dyes. For staining of $\mathrm{HS}$, cuprolinic blue is very useful. Under critical electrolyte concentration this cationic dye specifically stains HS and can therefore be used as a marker for GBM HS [29]. A recent study using cuprolinic blue demonstrated a reduction of $\mathrm{HS}$-associated anionic sites within the GBM in patients with diabetic nephropathy, which was inversely correlated with the degree of albuminuria [30]. A decrease of the number and density of cuprolinic blue anionic sites in the GBM of diabetic BB-rats has also been demonstrated [31]. In this study we analysed the HS-associated anionic sites in the GBM of STZ-diabetic rats with a pronounced albuminuria.

\section{Materials and methods}

Animals. Diabetes was induced in overnight-fasted male Wistar-Münich rats (Jackson Laboratory, Bar Harbor, Me., USA) of $150 \mathrm{~g}$ body weight by intravenous injection of $55 \mathrm{mg}$ $\mathrm{STZ} / \mathrm{kg}$ body weight, freshly prepared in $10 \mathrm{mmol} / \mathrm{l}$ citrate buffer $(\mathrm{pH} 4.5), 25 \mathrm{mg} / \mathrm{ml}$. Animals were treated three times a week (Monday, Wednesday, Friday at 17.00 hours) with a low dose (1.2 IU) of bovine insulin (Ultralente, Novo, Copenhagen, Denmark) to maintain blood glucose levels around $25 \mathrm{mmol} / \mathrm{l}$. Sex- and age-matched rats that did not receive STZ served as controls. The rats were followed-up monthly for body weight, blood glucose and albuminuria as described previously [32]. Blood glucose level was determined by Haemo-Glukotest 1-44R (Boehringer Mannhein, Mannheim, Germany) and albuminuria by rocket electrophoresis according to Laurell [33] using goat anti-rat albumin and rat albumin as the standard (both from Nordic, Tilburg, The Netherlands).

Tissue collection and cuprolinic blue staining. Eight months after induction of diabetes, rats were anaesthetized by Inactin (Byk Gulden, Konstanz, Germany); control rats, $140 \mathrm{mg} / \mathrm{kg}$ body weight; diabetic rats, $110 \mathrm{mg} / \mathrm{kg}$ body weight; kidneys were removed, decapsulated and weighed. Small cubes $\left(1 \mathrm{~mm}^{3}\right)$ of renal cortex tissue were fixed for $2 \mathrm{~h}$ by immersion fixation in lysine - periodate $-2 \%$ paraformaldehyde fixative, cryoprotected in $2.3 \mathrm{~mol} / \mathrm{l}$ sucrose for $1 \mathrm{~h}$, snap-frozen and stored in liquid nitrogen. To avoid penetration problems of the cationic dye, $30-\mu \mathrm{m}$ cryostat sections were cut, rinsed

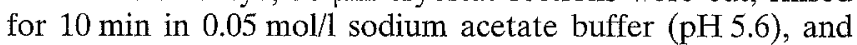
stained overnight at room temperature in 0.05 sodium acetate buffer ( $\mathrm{pH} 5.6$ ) containing $2 \%$ glutaraldehyde, $0.2 \mathrm{~mol} / 1$ $\mathrm{MgCl}_{2}$ and $0.2 \%$ cuprolinic blue (BDH Chemicals, Poole, Dorset, UK). Sections were rinsed three times for $10 \mathrm{~min}$ in the same buffer without cuprolinic blue, incubated for $1 \mathrm{~h}$ in a $1 \%$ sodium tungstate solution followed by an additional $30 \mathrm{~min}$ in $1 \%$ sodium tungstate in $50 \%$ ethanol, dehydrated in graded ethanol and embedded in Epon 812. Ultrathin sections were prepared on a LKB Ultratome, poststained for $45 \mathrm{~min}$ with uranyl acetate and examined in a JEOL 1200 EX II electron microscope (Jeol, Tokyo, Japan). Specificity of the cuprolinic blue staining for HS was controlled in two diabetic and two control rats by a $1-h$ preincubation (before cuprolinic blue staining) at $37^{\circ} \mathrm{C}$ of $30-\mu \mathrm{m}$ cryostat sections with $1 \mathrm{U} / \mathrm{ml}$ heparitinase from Flavobacterium heparinum (Seikagaku, Tokyo, Japan) in $0.1 \mathrm{~mol} / \mathrm{l}$ sodium acetate buffer containing $10 \mathrm{mmol} / \mathrm{l} \mathrm{Ca}^{++}(\mathrm{pH} \mathrm{7.0)}$, or with $0.1 \mathrm{U} / \mathrm{ml}$ chondroitinase ABC from Proteus vulgaris (Seikagaku) in $0.1 \mathrm{~mol} / 1$ Tris/ $\mathrm{HCl}(\mathrm{pH} \mathrm{8.0)}$, or the buffers alone without the enzymes.

Morphometric analysis. Thirty-five photomicrographs of randomly-chosen cross-sectioned glomerular capillary loops with a final magnification of $68,000 \times$ were taken from at least three randomly-chosen glomeruli per animal. The photomicrographs were analysed using a CCD RGB camera (Sony CA$325 \mathrm{AP}$ ) with a macro objective, attached to a VIDAS plus image analysis system (Kontron Inc., München, Germany). After the image was digitized, three lines were drawn interactively: the GBM was lined between the epithelial foot processes and the outer GBM and between the endothelial cells and the inner GBM. A third line was drawn just beneath the anionic sites in the lamina rara externa (LRE) of the GBM. The image was corrected for unequal lighting and background staining. A fixed threshold was applied to determine areas of positive staining. Since we observed that the anionic sites in the LRE were larger in size than those found in the remaining part of the GBM (lamina densa and lamina rara interna $(\mathrm{LD}+\mathrm{LRI})$ ), anionic site analysis was performed separately for the LRE and LD + LRI. From the resulting binary images of each photomicrograph the following parameters were calculated: a) the number of anionic sites per $\mu \mathrm{m}$ GBM; b) the mean anionic site surface $\left(\mathrm{nm}^{2}\right)$, which gives an impression of the mean surface (= area) of each individual anionic site; $\mathrm{c}$ ) the total anionic site surface $\left(\mathrm{nm}^{2} / \mu \mathrm{m}\right.$ GBM), defined as the number of anionic sites/ $\mu \mathrm{m}$ GBM multiplied with the mean anionic site surface $(a \times b)$; d) the thickness of the LRE and LD + LRI $(\mathrm{nm})$; and e) the relative anionic site surface $(\%)$. Latter parameter was calculated as follows: [(total anionic site surface LRE or LD + LRI/total surface LRE or LD + LRI) $\times 100 \%]$, 

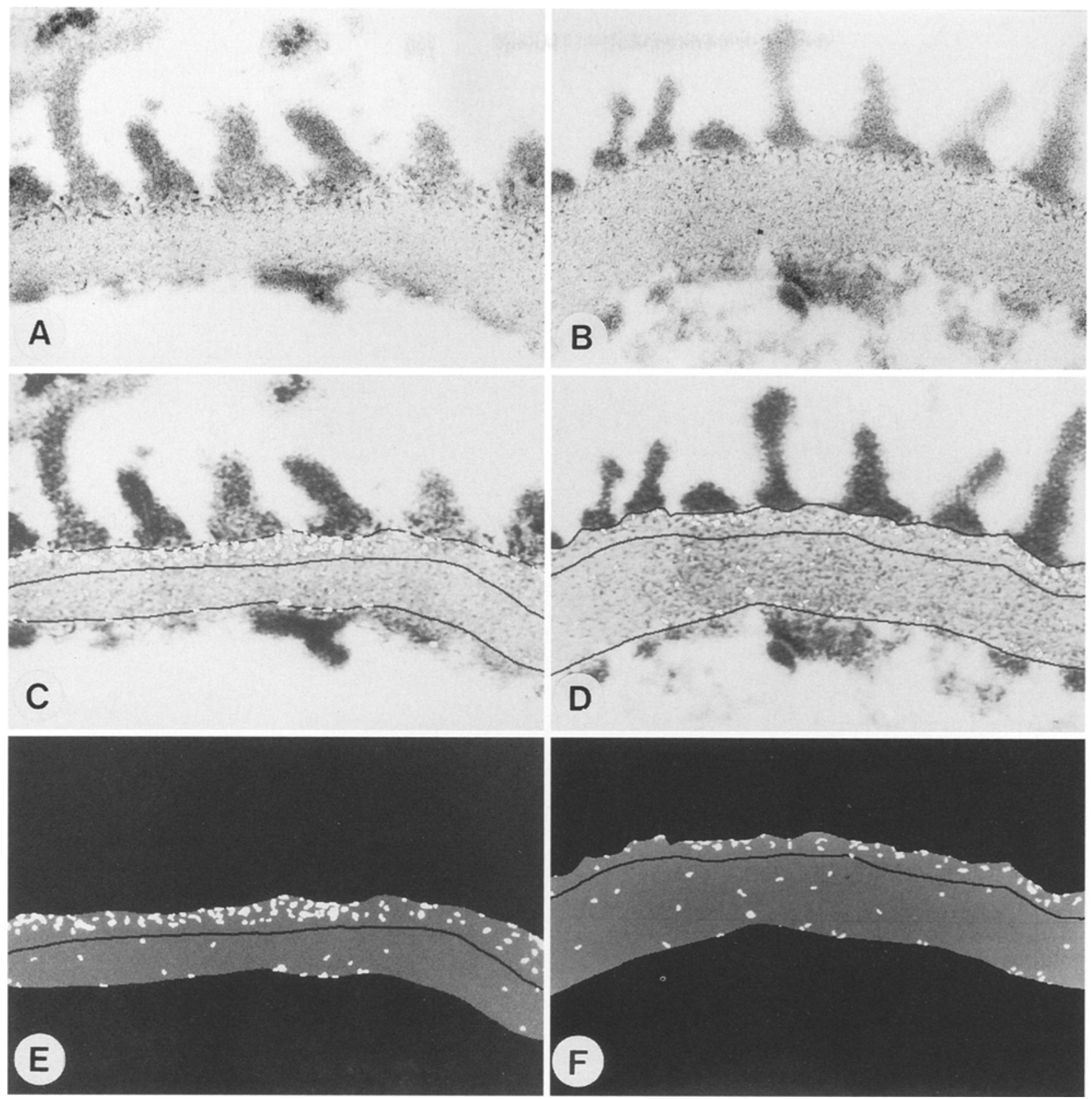

Fig. 1(A-F). Staining of the HS-associated anionic sites in the $\mathrm{GBM}$ of a control $(\mathbf{A}, \mathbf{C}, \mathbf{E})$ and a diabetic $(\mathbf{B}, \mathbf{D}, \mathbf{F})$ rat. Note that the majority of the anionic sites are localized in the LRE of the GBM. The original photomicrographs are seen in (A) and $(\mathbf{B})$. These images were digitized, three lines were drawn interactively as indicated in Methods, and the computerized anionic sites are shown in white $(\mathbf{C})$ and $(\mathbf{D})$. The corresponding binary images, on which the analysis was performed, are shown in (E) and (F). (Magnification: 50,000 $\times$ )

and expresses the percentage of the LRE or LD + LRI which is occupied by anionic sites. The analysis of the GBM anionic sites after pretreatment with glycosaminoglycan-degrading enzymes was also done on 35 photomicrographs from at least three randomly-chosen glomeruli/animal.

\section{Statistical analysis}

For statistical analysis the Mann-Whitney test was used for albuminuria, blood glucose concentration, body weight and kidney weight (Table 1 ). To analyse the morphometric data we used a special analysis of variance (ANOVA) approach, namely a single-factor nested design with random nesting factor, the single factor being presence/absence of diabetes, and animals as the nesting factor. $p$-values less than 0.05 were regarded as significant. Values are expressed as median values and (range).

\section{Results}

Development of diabetic nephropathy in STZ-diabetic rats. After a diabetes duration of 8 months an increased urinary albumin excretion developed and en- 
Table 1. Characteristics of the rats 8 months after induction of diabetes

\begin{tabular}{llll}
\hline Parameter & $\begin{array}{l}\text { Diabetic rats } \\
(n=8)\end{array}$ & $\begin{array}{l}\text { Control rats } \\
(n=5)\end{array}$ & $\begin{array}{l}p \text {-value } \\
\text { Mann-Whitney }\end{array}$ \\
\hline Albuminuria $(\mathrm{mg} / 24 \mathrm{~h})$ & $40.3(32.2-62.0)$ & $0.8(0.2-0.9)$ & $<0.002$ \\
Blood glucose $(\mathrm{mmol} / \mathrm{l})$ & $23.0(20.9-25.2)$ & $4.8(4.5-5.1)$ & $<0.002$ \\
Body weight $(\mathrm{g})$ & $228(212-256)$ & $370(368-391)$ & $<0.002$ \\
Wet kidney weight $(\mathrm{g})$ & $3.12(2.96-3.26)$ & $2.34(2.25-2.51)$ & $<0.002$ \\
\hline
\end{tabular}

Values are median (range)

Table 2. Influence of glycosaminoglycan-degrading enzymes on the number of anionic sites in the LRE or LD + LRI

\begin{tabular}{|c|c|c|c|c|c|c|}
\hline \multirow[t]{2}{*}{ Rat no. } & \multicolumn{2}{|c|}{ No pretreatment } & \multicolumn{2}{|c|}{ Heparitinase } & \multicolumn{2}{|c|}{ Chondroitinase $\mathrm{ABC}$} \\
\hline & $\overline{\mathrm{LRE}}$ & $\mathrm{LD}+\mathrm{LRI}$ & LRE & $\mathrm{LD}+\mathrm{LRI}$ & LRE & $\mathrm{LD}+\mathrm{LRI}$ \\
\hline \multicolumn{7}{|l|}{$\overline{\text { Diabetic }}$} \\
\hline 2 & 32 & 9 & 3 & 2 & 37 & 10 \\
\hline \multicolumn{7}{|l|}{ Control } \\
\hline 1 & 37 & 8 & 2 & 1 & 34 & 6 \\
\hline
\end{tabular}

Table 3. Analysis of the HS-associated anionic sites of the GBM

\begin{tabular}{|c|c|c|c|}
\hline Parameter & $\begin{array}{l}\text { Diabetic rats } \\
(n=8)\end{array}$ & $\begin{array}{l}\text { Control rats } \\
(n=5)\end{array}$ & $\begin{array}{l}p \text {-value } \\
\text { ANOVA }\end{array}$ \\
\hline \multicolumn{4}{|l|}{ Lamina rara externa (LRE) } \\
\hline Number of anionic sites (sites/ $\mu \mathrm{m}$ GBM) & $34(25-42)$ & $42(39-46)$ & $<0.02$ \\
\hline Total anionic site surface $\left(\mathrm{nm}^{2} / \mu \mathrm{m}\right.$ GBM$)$ & $4957(2975-7056)$ & $6670(5838-8580)$ & $<0.02$ \\
\hline Thickness LRE (nm) & $76(63-88)$ & $74(72-86)$ & NS \\
\hline Relative anionic site surface (\%) & $6.79(4.64-8.82)$ & $9.31(7.48-12.15)$ & $<0.03$ \\
\hline Surface/anionic site $\left(\mathrm{nm}^{2}\right)$ & $66(57-82)^{a}$ & $62(60-79)^{b}$ & NS \\
\hline Total anionic site surface $\left(\mathrm{nm}^{2} / \mu \mathrm{m}\right.$ GBM$)$ & $895(195-1148)$ & $711(600-936)$ & NS \\
\hline Thickness LD + LRI (nm) & $208(166-242)$ & $162(144-186)$ & $<0.01$ \\
\hline Relative anionic site surface (\%) & $0.45(0.14-0.64)$ & $0.44(0.37-0.53)$ & NS \\
\hline
\end{tabular}

Values are median (range)

${ }^{a} \mathrm{LD}+\mathrm{LRI}$ vs LRE, $p<0.001 ;{ }^{\mathrm{b}} \mathrm{LD}+\mathrm{LRI}$ vs LRE, $p<0.01$

largement of the kidneys, which are both characteristic for diabetic nephropathy (Table 1). The diabetic animals were hyperglycaemic during the entire study period as controlled by monthly blood glucose monitoring. The blood glucose values in Table 1 correspond to the blood glucose levels measured just before the animals were killed. Table 1 also shows that the diabetic rats gained less weight compared to control rats. Light microscopy demonstrated a moderate increase of the mesangial matrix in the glomeruli of most diabetic rats as described previously [32].

Analysis of the HS-associated anionic sites in the $G B M$. The cuprolinic blue staining procedure yielded a homogeneously-stained thin layer of anionic sites within the LRE of the GBM. These sites represent, in the diabetic rats, $74 \%$ and in the control rats, $81 \%$ of the total number of anionic sites within the GBM. The other anionic sites were scattered throughout the LD + LRI (Fig. 1 A, B). Figure 1 also demonstrates the three lines which indicate the epithelial and endothelial borders of the GBM and which mark the layer of anionic sites in the LRE (Fig. 1C,D). The corresponding binary images are shown in Figure 1 (E,F). The HS-specificity of the staining was demonstrated by pretreatment of sections originating from two diabetic and two control rats with glycosaminoglycan-degrading enzymes. As can be seen in Table 2, pretreatment of the sections with heparitinase (an enzyme that specifically cleaves HS) reduced the number of anionic sites in the LRE and LD + LRI to background levels, whereas chondroitinase ABC (an enzyme that specifically cleaves chondroitin sulphate $\mathrm{A}$ and $\mathrm{C}$ and dermatan sulphate) had hardly any effect on the number of anionic sites in the LRE and LD + LRI. Preincubation of the sections with acetate or. Tris buffer alone (control buffers for the glycosaminoglycan-degrading enzymes) had no influence on the number of anionic sites in both LRE and LD + LRI (not 
shown). This indicates that in both the diabetic and control rats the GBM anionic sites are HS-associated.

The results of the analysis of the anionic sites are shown in Table 3. The number of anionic sites in the LRE was significantly reduced in the diabetic rats by $19 \%$ compared to the control animals $(p<0.02)$. Since it has been suggested that the size of the anionic sites gives information about the proteoglycan structure [29], we also measured the mean anionic site surface in every individual rat. Although the mean anionic site surface in the LRE was not significantly different between both groups, there was a tendency towards smaller sites in the diabetic rats. The total anionic site surface in the LRE was reduced by $26 \%(p<0.02)$, the relative anionic site surface in the LRE was significantly decreased in the diabetic rats by $27 \%$ compared to control rats $(p<0.03)$, which was not due to a difference in the thickness of the LRE. These data suggest a reduced HS-associated charge barrier within the LRE in the diabetic rats. A minority of anionic sites were found in the LD + LRI in both diabetic and control rats. Latter sites were significantly smaller than the anionic sites in the LRE $(p<0.001$ for diabetic rats and $p<0.01$ for control rats). Consequently, lower values compared to the LRE were found for both the total and relative anionic site surface (Table 3 ). From these data it can be calculated that 15 or $10 \%$ of the total anionic site surface in the GBM is located in the LD + LRI for the diabetic or control rats, respectively. This suggests, using this technique, that the HS-associated anionic sites in the LRE are the major determinants for GBM charge.

Since all diabetic animals were only analysed 8 months after diabetes induction, we did not find a correlation between the different anionic site parameters and albuminuria. This is probably due to the fact that the analysis was done only at one time point ( 8 months) and not also at earlier time points.

\section{Discussion}

In this study the HS-associated anionic sites in the GBM of albuminuric STZ-diabetic rats and control rats were analysed using the cationic dye cuprolinic blue. It has been documented that under critical electrolyte conditions, this dye specifically stains HS in extracellular matrix [29], as also revealed by our control experiments with diabetic and control animals using glycosaminoglycan-degrading enzymes.

We found in this STZ-induced diabetic nephropathy model and in normal rats that the majority of the HS-associated anionic sites are localized in the LRE of the GBM. This was also found by Vernier et al. [30] in the kidneys of insulin-dependent diabetic patients and by Chakrabarti et al [31] in the kidneys of diabetic BB-rats. The results show that in the LRE of diabetic rats the number of anionic sites, the total anionic site surface and the relative anionic site surface were significantly decreased compared to control rats. These differences cannot be explained by differences in the LRE width, since these were equal in both groups. These results strongly suggest a reduced HS-associated charge barrier within the LRE in experimental diabetic nephropathy. No alterations were observed for the anionic sites in the LD + LRI of the GBM. The number of anionic sites in the LD + LRI represents 26 and $19 \%$ of the total number of anionic sites in the GBM for diabetic and control rats, respectively. Furthermore, the mean surface per anionic site in the LD + LRI is significantly smaller than in the LRE. Since it has been suggested that the size of the anionic sites gives some information about the structure of the HSPG molecule [29], this analysis suggests that two types of GBM HS are present. The major population is identified as larger anionic sites in the LRE which are subject to alterations in diabetes, and a minor population, recognized as smaller anionic sites in the LD + LRI, which remain unaltered in diabetes. In a previous study [34], we suggested the existence of at least two different types of basement membrane HSPG, based on differences in glomerular staining pattern. Using a monoclonal antibody against the core protein of the large EHS-HSPG we observed strong mesangial and weak GBM staining, whereas monoclonal antibodies against the core protein of GBM HSPG yielded weak mesangial and strong GBM staining. Whether these two different HSPG populations are related to the two different HS populations as found in the present study is not known. Furthermore, no data are available whether the expression of these two different types of HSPG in diabetes are regulated separately and differently. At the same time, one should realize that histological processing can markedly influence the localization of HS-associated anionic sites in basement membranes, as shown by Chan et al. [35]. By changing the fixative from glutaraldehyde to formaldehyde the cuprolinic blue HS-associated anionic sites and the HSPG-core protein (visualized by the immunogold technique) were found to co-localize over the LD of basement membranes. This observation later led to the suggestion that the lamina lucidae/rarae of basement membranes were artefacts, and that HSPG is localized throughout the whole GBM [36]. This might indicate that the localization of HS-associated anionic sites in the LRE is due to redistribution during histological processing. Even if this is true, the total anionic site surface of all GBM anionic sites (LRE + LD + LRI) still decreased by $21 \%(p<0.02)$ and the relative anionic site surface of all GBM anionic sites decreased even by $26 \%$ as a consequence of the increased thickness of the $\operatorname{GBM}(p<0.002)$. Therefore, irrespective of eventual redistribution of the HS-associated anionic 
sites due to histological processing, our results indicate a reduced HS-associated charge barrier in the GBM of long-term diabetic rats, that might be explained by a reduced number of HSPG molecules in the GBM and/or an altered structure; e.g., reduced charge of HS.

In a previous study on isolated glomeruli of diabetic rats 8 months after STZ injection, no absolute difference in GBM HS content was found between diabetic and control rats [32]. The HS measurements of that study were done on glomerular extracts with an inhibition-ELISA, using anti-HS monoclonal antibody JM-403, specific for GBM HS [9]. Combining the results of the present study with those of the study mentioned above supports the idea of alterations in the structure of GBM HS. The most likely explanation for this is a reduction of the sulphation of GBM HS in diabetic rats. Undersulphation of HS both from the GBM [24] as well as from other tissues $[37,38]$ in STZ rats has been reported. Recently, it has been shown that culture of mesangial cells on non-enzymatic glycated mesangial matrix or prolonged exposure to elevated glucose levels leads to the production of undersulphated proteoglycans [39, 40]. HS sulphation occurs in the Golgi-apparatus of the producing cell and is determined by the key enzyme glucosaminyl $\mathrm{N}$-deacetylase [41]. A decreased activity of this enzyme has been found in STZ-diabetic rats [42-44]. Finally, at present the influence of other diabetes-related factors such as non-enzymatic glycation on the staining properties of cuprolinic blue in diabetic GBM is not known. Non-enzymatic glycation of intrinsic GBM constituents and the cross-linking of non-enzymatic glycated serum proteins to the GBM leads to an altered three-dimensional architecture of the GBM $[18,45]$, that might interfere with the staining of the HS-associated anionic sites. In conclusion, this study demonstrates a reduced HS charge barrier in the LRE of the GBM of rats with STZ-induced diabetes, which might explain the increased urinary albumin excretion found in these animals.

Acknowledgements. This study was supported by a grant (C 90.1040) from the Dutch Kidney Foundation. The Nephrology Department participates in a concerted action "Alterations in extracellular matrix components in diabetic nephropathy and other glomerular diseases" which is financially supported by the EC within the Biomed I program (BMH1CT92-1766). The authors thank Mr. J.Koedam and Mr. G. Grutters of the Central Animal Laboratory for their assistance in the animal experiments, and Ir. T.de Boo (Department of Medical Statistics) for his expert help in the statistical analysis.

\section{References}

1. Kanwar YS, Liu ZZ, Kashihara N, Wallner EI (1991) Current status of the structural and functional basis of glomerular filtration and proteinuria. Semin Nephrol 11: 390-413

2. Cotran RS, Rennke HG (1983) Anionic sites and the mechanisms of proteinuria. New Engl J Med 309: 1050-1052

3. Farquhar MG (1991) The glomerular basement membrane. A selective macromolecular filter. In: Hay ED (ed) Cell biology of extracellular matrix. Plenum Press, New York, pp 365-418

4. Rosenzweig LJ, Kanwar YS (1982) Removal of sulfated (heparan sulfate) or nonsulfated (hyaluronic acid) glycosaminoglycans results in increased permeability of the glomerular basement membrane to ${ }^{125} \mathrm{I}$-bovine serum albumin. Lab Invest 47: 177-184

5. Kanwar YS, Linker A, Farquhar MG (1980) Increased permeability of the glomerular basement membrane to ferritin after removal of glycosaminoglycans (heparan sulfate) by enzyme digestion. J Cell Biol 86: 680-693

6. Vehaskari VM, Root ER, Germuth FG, Robson AM (1982) Glomerular charge and urinary protein excretion. Effects of systemic and intrarenal polycation infusion in the rat. Kidney Int 22: 127-135

7. Hunsicker LG, Shearer TP, Shaffer SJ (1981) Acute reversible proteinuria induced by infusion of the polycation hexadimethrine. Kidney Int 20:7-17

8. Kelley VE, Cavallo T (1978) Glomerular permeability, transfer of native ferritin in glomeruli with decreased anionic sites. Lab Invest 39: 547-553

9. Born van den J, Heuvel van den LPWJ, Bakker MAH, Veerkamp JH, Assmann KJM, Berden JHM (1992) A monoclonal antibody against GBM heparan sulfate induces an acute selective proteinuria in rats. Kidney Int 41: 115-123

10. Mogensen CE (1984) Microalbuminuria predicts clinical proteinuria and early mortality in maturity-onset diabetes. New Engl J Med 310: 356-360

11. Viberti GC, Hill RD, Jarrett RJ, Argyropoulos A, Mahmud U, Keen H (1982) Microalbuminuria as a predictor of clinical nephropathy in insulin-dependent diabetes mellitus. Lancet 1: 1430-1432

12. Deckert T, Feldt-Rasmussen B, Djurup R, Deckert M (1988) Glomerular size and charge selectivity in insulin-dependent diabetes mellitus. Kidney Int 33: 100-106

13. Deckert T, Kofoed-Enevoldsen A, Vidal P, Nørgaard K, Andreasen HB, Feldt-Rasmussen B (1993) Size- and charge selectivity of glomerular filtration in type 1 (insulin-dependent) diabetic patients with and without albuminuria. Diabetologia 36: 244-251

14. Bangstad H-J, Kofoed-Enevoldsen A, Dahl-Jørgensen K, Hanssen KF (1992) Glomerular charge selectivity and the influence of improved blood glucose control in type 1 (insulin-dependent) diabetic patients with microalbuminuria. Diabetologia 35: 1165-1169

15. Kverneland A, Feldt-Rasmussen B, Vidal P et al. (1986) Evidence of changes in renal charge selectivity in patients with type 1 (insulin-dependent) diabetes mellitus. Diabetologia 29: 634-639

16. Parthasarathy N, Spiro RG (1982) Effect of diabetes on the glycosaminoglycan component of the human glomerular basement membrane. Diabetes 31: 738-741

17. Shimomura H, Spiro RG (1987) Studies on macromolecular components of human glomerular basement membrane and alterations in diabetes; decreased levels of heparan sulfate proteoglycan and laminin. Diabetes 36: 374-381

18. Makino H, Yamasaki Y, Haramoto T et al. (1993) Ultrastructural changes of extracellular matrices in diabetic 
nephropathy revealed by high resolution scanning and immunoelectron microscopy. Lab Invest 68: 45-55

19. Saraswathi S, Vasan NS (1983) Alterations in the rat renal glycosaminoglycans in streptozotocin-induced diabetes. Biochim Biophys Acta 755: 237-243

20. Wu V-Y, Wilson B, Cohen MP (1987) Disturbances in glomerular basement membrane glycosaminoglycans in experimental diabetes. Diabetes 369: 679-683

21. Reddi AS (1991) Prevention of albuminuria by captopril in diabetic rats. Gen Pharmacol 22: 323-328

22. Reddi AS, Ramamurthi R, Miller M, Dhuper S, Lasker N (1991) Enalapril improves albuminuria by preventing glomerular loss of heparan sulfate in diabetic rats. Biochem Med Metab Biol 45: 119-131

23. Templeton DM (1989) Retention of glomerular basement membrane-proteoglycans accompanying loss of anionic site staining in experimental diabetes. Lab Invest 61: 202211

24. Cohen MP, Klepser H, Wu V-Y (1988) Undersulfation of glomerular basement membrane heparan sulfate in experimental diabetes and lack of correction with aldolase reductase inhibition. Diabetes 37: 1324-1327

25. Born van den J, Heuvel van den LPWJ, Bakker MAH et al. (1993) Distribution of GBM heparan sulfate proteoglycan core protein and side chains in human glomerular diseases. Kidney Int 43: 454-463

26. Tamsma JT, Born van den J., Bruijn JA et al. (1994) Expression of glomerular extracellular matrix components in human diabetic nephropathy: decrease of heparan sulphate in the glomerular basement membrane. Diabetologia 37: $313-320$

27. Nerlich A, Schleicher E (1991) Immunohistochemical localization of extracellular matrix components in human diabetic glomerular lesions. Am J Pathol 139: 889-899

28. Makino H, Ikeda S, Haramoto T, Ota Z (1992) Heparan sulfate proteoglycans are lost in patients with diabetic nephropathy. Nephron 61: 415-421

29. Kuppevelt van THMSM, Veerkamp JH (1994) Application of cationic probes for the ultrastructural localization of proteoglycans in basement membranes. Microsc Res Tech 28: $125-140$

30. Vernier RL, Steffes MW, Sisson-Ross S, Mauer M (1992) Heparan sulfate proteoglycan in the glomerular basement membrane in type I diabetes mellitus. Kidney Int 41: 1070-1080

31. Chakrabarti S, Ma N, Sima AAF (1989) Reduced number of anionic sites is associated with glomerular basement membrane thickening in the diabetic BB-rat. Diabetologia 32: $826-828$

32. Born van den J, Kraats van AA, Bakker MAH et al. (1995) Selective proteinuria in diabetic nephropathy in the rat is associated with a relative decrease in glomerular basement membrane heparan sulphate. Diabetologia 38: 161172
33. Laurell CB (1966) Quantitative estimation of proteins of electrophoresis in agarose gel containing antibodies. Anal Biochem 15: 45-52

34. Born van den J, Heuvel van den LPWJ, Bakker MAH, Veerkamp JH, Assmann KJM, Berden JHM (1994) Monoclonal antibodies against the core protein and glycosaminoglycan side chain of glomerular basement membrane heparan sulfate proteoglycan: characterization and immunohistochemical application in human tissues. J Histochem Cytochem 42: 89-102

35. Chan FL, Inoue S, Leblond CP (1992) Localization of heparan sulfate proteoglycan in basement membrane by side chain staining with cuprolinic blue as compared with core protein labeling with immunogold. J Histochem Cytochem 40: 1559-1572

36. Chan FL, Inoue S, Leblond CP (1992) Localization of heparan sulfate proteoglycan in basement membranes. $\mathrm{J}$ Histochem Cytochem 40: 1807-1808

37. Levy P, Picard J, Bruel A (1984) Evidence for diabetes-induced alterations in the sulfation of heparan sulfate intestinal epithelial cells. Life Sci 35: 2613-2620

38. Kjellén L, Bielefeld D, Höök M (1983) Reduced sulfation of liver heparan sulfate in experimentally diabetic rats. Diabetes 32: 337-342

39. Silbiger S, Crowley S, Shan Z, Brownlee M, Santriano J, Schlondorff D (1993) Nonenzymatic glycation of mesangial matrix and prolonged exposure of mesangial matrix to elevated glucose reduces collagen synthesis and proteoglycan charge. Kidney Int 43: 853-864

40. Silbiger S, Schlondorff D, Crowley S et al. (1993) The effect of glucose on proteoglycans produced by cultured mesangial cells. Diabetes 42: 1815-1822

41. Lindahl U, Kusche M, Lidholt K, Oscarsson L-G (1989) Biosynthesis of heparin and heparan sulfate. Ann NY Acad Sci 556: 36-50

42. Unger E, Pettersson I, Eriksson UJ, Lindahl U, Kjellén L (1991) Decreased activity of the heparan sulfate-modifying enzyme glucosaminyl $\mathrm{N}$-deacetylase in hepatocytes from streptozotocin-diabetic rats. J Biol Chem 266: 86718674

43. Kofoed-Enevoldsen A (1992) Inhibition of glomerular glucosaminyl N-deacetylase in diabetic rats. Kidney Int 41 : 763-767

44. Kofoed-Enevoldsen A, Noonan D, Deckert T (1993) Diabetes mellitus induced inhibition of glucosaminyl N-deacetylase: effect of short-term blood glucose control in diabetic rats. Diabetologia 36: 310-315

45. Anderson SS, Tsilibary EC, Charonis AS (1993) Nonenzymatic glycosylation-induced modifications of intact bovine kidney tubular basement membrane. J Clin Invest 92: 3045-3052 